\title{
Theoretical characterization of the lowest-energy absorption band of pyrrole
}

\author{
Björn O. Roos, Per-Åke Malmqvist, and Vincent Molina \\ Department of Theoretical Chemistry, Lund University, Chemical Center, P.O.B. 124, S-221 00 Lund, \\ Sweden \\ Luis Serrano-Andrés ${ }^{a)}$ and Manuela Merchán \\ Departamento de Química Física, Instituto de Ciencia Molecular, Universitat de València, Dr. Moliner 50, \\ Burjassot, E-46100 Valencia, Spain
}

(Received 22 October 2001; accepted 6 February 2002)

\begin{abstract}
The lowest-energy band of the electronic spectrum of pyrrole has been studied with vibrational resolution by using multiconfigurational second-order perturbation theory (CASPT2) and its multistate extension (MS-CASPT2) in conjunction with large atomic natural orbital-type basis sets including Rydberg functions. The obtained results provide a consistent picture of the recorded spectrum in the energy region 5.5-6.5 eV and confirm that the bulk of the intensity of the band arises from a $\pi \pi^{*}$ intravalence transition, in contradiction to recent theoretical claims. Computed band origins for the $3 s, 3 p$ Rydberg electronic transitions are in agreement with the available experimental data, although new assignments are suggested. As illustrated in the paper, the proper treatment of the valence-Rydberg mixing is particularly challenging for $a b$ initio methodologies and can be seen as the main source of deviation among the recent theoretical results as regards the position of the low-lying valence excited states of pyrrole. (C) 2002 American Institute of Physics.
\end{abstract} [DOI: 10.1063/1.1465406]

\section{INTRODUCTION}

Multiconfigurational second-order perturbation theory based on a complete active space (CAS) self-consistent field (SCF) reference wave function, the CASSCF second-order perturbation theory, CASPT2 method, has proven to be a reliable method to treat a large variety of electronic structure problems in molecular systems. ${ }^{1,2}$ Since the method was proposed a decade ago, close to 200 compounds ranging from basic organic molecules to large transition metal complexes and biomolecules have been successfully studied (for a selected number of applications see, for instance, recent reviews. ${ }^{3-6}$ ) The study on the electronic spectrum of pyrrole and related heterocycles published in $1993^{7}$ can be found among the earliest applications in electronic spectroscopy that used the CASPT2 method. The CASPT2 results for pyrrole did drastically differ from previous theoretical results but were found to be in agreement with experimental data (see Refs. 7 and 8). Four theoretical studies have been subsequently performed at different levels of theory. Overall, the findings reported by Nakano et al., ${ }^{9}$ Trofimov and Schirmer, ${ }^{10}$ Palmer et al., ${ }^{11}$ and Christiansen et al. ${ }^{12}$ agree with the assignments made by us in 1993 based on the CASPT2 results. ${ }^{7}$ There are, however, certain discrepancies between the CASPT2 and those results, mainly concerning the assignments related to the observed lowest-energy band of the electronic absorption spectrum of pyrrole, worth examining in detail. In particular, the key issue of controversy is whether the lowest valence excited state of ${ }^{1} B_{2}$ symmetry contributes to the lowest-energy band or not.

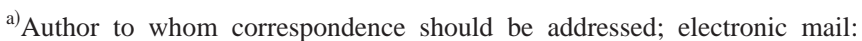
luis.serrano@uv.es
}

The experimental absorption spectrum of pyrrole has two intense bands ${ }^{11,13-19}$ (see previous papers for earlier references). ${ }^{7,14-16}$ The lowest-energy band has an intensity maximum near $6.0 \mathrm{eV}$ while the second energy has it near $7.5 \mathrm{eV}$. That most of the intensity of the latter can be attributed to the presence of high-lying valence excited states has been supported both experimentally ${ }^{11,15-18}$ and theoretically. $^{7,9-12}$ In the same energy range a number of Rydberg transitions have also been identified. There is agreement between theory and experiment regarding the nature and placement of the lowest singlet excited state of pyrrole. The character of the peak detected at $5.22 \mathrm{eV}^{13,15-17}$ has been clearly determined to be of Rydberg nature, corresponding to a transition to the $1{ }^{1} A_{2}(3 s)$ Rydberg state. The dipole-forbidden character of the lowest electronic transition in pyrrole was firmly established in our previous work ${ }^{7}$ and the assignment was supported by most of the previous ${ }^{7,20-22}$ and most recent theoretical studies, ${ }^{9-12}$ in agreement with earlier analyses. ${ }^{15,16}$ However, for the lowest-energy band recorded in the energy range $5.5-6.5 \mathrm{eV}$ one can find in the theoretical literature (not in the experimental one) as many arguments in favor of the Rydberg character of the band as about its valence nature (for historical details the reader is referred to previous references). ${ }^{7,10-12,15}$ In the traditional interpretation of the pyrrole spectrum, ${ }^{15}$ the intensity of the lowestenergy band is associated with the presence in this region of the ${ }^{1} B_{2}$ valence excited state of $\pi \pi^{*}$ character. Of course, a number of low-lying Rydberg transitions are also expected to be interleaved in the same energy region, as has been confirmed both experimentally and theoretically. Indeed, the simultaneous occurrence of many overlapping Rydberg series and valence excited states is responsible for the complex 
structure of the electronic spectrum of pyrrole. It is also the underlying reason why the theoretical calculations become especially challenging. In order to elucidate whether a physical valence-Rydberg mixing takes place or it is just an artifact of the calculation seems to be the crucial issue for the $a b$ initio research of excited states. It particularly holds true for the two lowest excited states of pyrrole of ${ }^{1} B_{2}$ symmetry. The previous CASPT2 results $^{7}$ vertically located the ${ }^{1} B_{2}(3 p)$ and ${ }^{1} B_{2}\left(\pi \pi^{*}\right)$ excited states at 5.78 and $6.00 \mathrm{eV}$, respectively. The computed oscillator strengths for the transitions were in agreement with the corresponding Rydberg and valence nature, 0.040 and 0.125 , respectively. Therefore, the CASPT2 results supported the traditional interpretation of the lowest-energy band of the absorption spectrum of pyrrole. Nevertheless, the recent large-scale calculations carried out with the multireference perturbation (MRMP) theory ${ }^{9}$ and the best estimate obtained using coupled-cluster (CC) response methodology ${ }^{12}$ place the ${ }^{1} B_{2}\left(\pi \pi^{*}\right)$ valence excited state at 6.51 and $6.57 \mathrm{eV}$, respectively. Time dependentdensity functional theory (TD-discrete Fourier transform DFT) $(\mathrm{HCTH})$ calculations $^{23}$ gave a similar picture, with a transition to the ${ }^{1} B_{2}\left(\pi \pi^{*}\right)$ valence state at $6.45 \mathrm{eV}$ and an oscillator strength smaller for the valence than for the Rydberg transition. The multireference configuration interaction (CI) result for the ${ }^{1} B_{2}\left(\pi \pi^{*}\right)$ state is somewhat higher (6.77 $\mathrm{eV}) .{ }^{11}$ That is, the difference from the CASPT2 result is as much as $0.6 \mathrm{eV}$. A considerable valence-Rydberg mixing has been reported for the lowest ${ }^{1} B_{2}$ valence excited state at both levels of calculation. Accordingly, the associated electronic transitions are predicted with too small oscillator strengths of comparable magnitude. It is appropriate to recall that at around $6.5 \mathrm{eV}$ the observed absorption spectrum in the vapor dips gently before the second-energy band begins to develop. ${ }^{11,15,17}$ Moreover, the traditional interpretation of the lowest-energy band as containing the valence ${ }^{1} B_{2}\left(\pi \pi^{*}\right)$ intensity is consistent with the experimental fact that the band is clearly identified both in vapor and condensed phases. The maximum of a clearly observed band has been measured in hexane at $5.96 \mathrm{eV}^{19}$ and in liquid pyrrole at $5.90 \mathrm{eV} .{ }^{16}$ In the crystal spectrum the band is placed at $6.0 \mathrm{eV}$, while a fourtimes stronger band, presumably corresponding to the $7.5 \mathrm{eV}$ valence transition in the vapor, has its maximum higher than the upper limit of measurement, $6.7 \mathrm{eV} .{ }^{16}$ The Rydberg states should play a minor role in condensed phases, therefore it would be hardly understandable if the band found in solution and crystal is not the consequence of an intravalence transition. Recently, Palmer et al. ${ }^{11}$ have expressed a similar opinion in their combined experimental-theoretical paper. They have carried out a reinvestigation and extension of the observed vacuum ultraviolet and near-threshold electron energy-loss (EEL) spectra. Despite the fact that their multireference CI result places the valence ${ }^{1} B_{2}$ state too high, they think that there can be little doubt from both experiment and theory that most of the intensity in the lowest-energy band arises from an intravalence electronic transition. ${ }^{11}$

The importance of the pyrrole system in many aspects of current chemistry (organic synthesis, biochemistry, conducting polymers, etc), together with the mentioned discrepancies of recent studies with respect to the CASPT2 results in connection with the assignment of the lowest-energy band has inspired us to undertake a more detailed study of the 5.5-6.5 eV energy region at the CASPT2 $2^{4,24}$ and multistate CASPT2 (MS-CASPT2) levels. ${ }^{25}$ As demonstrated in the presentation of the method and in applications performed subsequently, the MS-CASPT2 level is able to handle spurious valence-Rydberg mixings. ${ }^{25-29}$ The geometry of the relevant excited states has been optimized at the CASPT2 level. In total six excited states have been considered. They are involved in the two lowest-energy $\pi \pi^{*}, 3 s$, and $3 p$ Rydberg electronic transitions. In addition to the vertical excitation energies, the corresponding band origins have been computed. The study includes zero point energy (ZPE) corrections. For the vibrational analysis CASPT2 force fields are employed to compute the corresponding vibronic intensities. The computed band including the contributions from the six transitions nicely match the absorption spectrum in the energy region of interest and confirms our previous assignments. As discussed below, an inadequate treatment of the valence-Rydberg mixing at the MRMP and CC levels is probably the major reason for the too high excitation energies computed for the lowest valence excited state of ${ }^{1} B_{2}$ symmetry. This problem is shared by the CASPT2 method in particular circumstances, as it will be commented, because the valence-Rydberg mixing is extremely sensitive to small changes in the level of the calculation, such as modifications in geometry, basis sets, active spaces, etc.

\section{METHODS AND COMPUTATIONAL DETAILS}

Generally contracted basis sets of atomic natural orbital (ANO) type obtained from the $\mathrm{C}, \mathrm{N}(14 s 9 p 4 d) / \mathrm{H}(8 s)$ primitive sets, ${ }^{30}$ the so-called ANO- $L$, with the $\mathrm{C}, \mathrm{N}[4 s 3 p 2 d] / \mathrm{H}[3 s 2 p]$ contraction schemes were used. They were supplemented with diffuse functions placed at the charge centroid of the ground state of the positive ion, contracted from a set of $8 s 8 p 8 d$ primitive functions. Two contraction sets of Rydberg functions were employed: $1 s 1 p$ and $1 s 1 p 1 d$. The former was used in the geometry optimizations and the latter for the computation of the vertical excitations and band origins. The Rydberg functions were built following the procedure described elsewhere. ${ }^{3}$ The pyrrole molecule, located in the $y z$ plane with the $z$ axis being the $\mathrm{C}_{2}$ symmetry axis, was computed within the $\mathrm{C}_{2 v}$ symmetry constraints. Geometry optimizations were also restricted to the $\mathrm{C}_{2 v}$ symmetry. Force field calculations, however, were performed in lower symmetries in order to compute the different components.

The reference wave functions and the molecular orbitals were obtained from state-average (SA)-CASSCF calculations, including all the states of interest for a given symmetry. For the geometry optimizations the active space comprises the $\pi$ valence molecular orbitals (valence excited states) plus the Rydberg orbitals as appropriate to describe the Rydberg states of interest. The active space including the five valence orbitals of $\pi$ character plus the nine $(n=3)$ Rydberg orbitals (six active electrons) was subsequently employed to compute transition energies and the remaining spectroscopic properties. The CASSCF wave functions were 
employed as reference functions in a single-state secondorder perturbation CASPT2 treatment. ${ }^{1,2}$ The coupling of the CASSCF wave functions via dynamic correlation was dealt with by means of the extended multistate CASPT2 approach, the MS-CASPT2 method. ${ }^{25}$ An effective Hamiltonian matrix is constructed where the diagonal elements correspond to the CASPT2 energies and the off-diagonal elements introduce the coupling to second order in the dynamic correlation energy. In this manner, the states considered in a MSCASPT2 computation can be treated simultaneously with the correlation effects on the reference functions included, and the possibly erroneous valence-Rydberg mixing can be removed. Well-known examples of a strong valence-Rydberg interaction at the CASSCF level are the excited states of ethene and butadiene. ${ }^{25,31} \mathrm{~A}$ similar situation occurs in $s$-tetrazine ${ }^{26}$ and $n$-tetrasilane. ${ }^{27}$ Perturbation modified CAS (PMCAS-CI) reference functions (the model states), ${ }^{25}$ i.e., linear combinations of all CAS states involved in the MSCASPT2 calculation, were employed to compute the corresponding transition dipole moments according to the CAS state interaction (CASSI) protocol. ${ }^{32,33}$

Geometry optimizations were carried out at the CASPT2 level for the seven states under consideration. It is worth mentioning that vibrational progressions were found to be particularly well described and in agreement with experimental data, in both intensities and energies, when CASPT2 optimized geometries were employed for the theoretical determination of the ${ }^{1} B_{2 u}$ and ${ }^{1} B_{1 u}$ vibronic bands of benzene. ${ }^{34}$ The band origins ( $0-0$ transition energies) were computed at the MS-CASPT2 level at the CASPT2 geometry minima of the corresponding states and include the zeropoint vibrational correction. One exception is the $2{ }^{1} A_{1}$ valence excited state, where a CASSCF force field was employed. The state is calculated to have many imaginary out-of-plane frequencies at the CASPT2 level. Because of the low intensity of this transition, its contribution to the final spectrum is, however, limited.

Regarding the calculations on the vibrational profiles for the absorption transitions, frequencies are obtained from harmonic analyses of the computed force fields. Intensities are obtained as oscillator strengths of the transitions, calculated as

$$
f=\frac{2}{3} \Delta E M_{g i, f j}^{2},
$$

where $\Delta E$ is the transition energy and $M_{g i, f j}^{2}$ the vibronic transition moment. The matrix elements describing the transition moment function are expressed in terms of FranckCondon factors, that is, overlaps between two sets of harmonic oscillator functions representing the force fields of the participating states. ${ }^{35}$ The vibronic transition moment is computed as

$$
\begin{aligned}
M_{g i, f j}= & M_{g f}\left(Q_{0}\right)\left\langle\phi_{i}(Q) \mid \phi_{j}(Q)\right\rangle \\
& +\sum_{k}\left(\frac{\partial M_{g f}}{\partial Q_{k}}\right)\left\langle\phi_{i}(Q)\left|Q_{k}\right| \phi_{j}(Q)\right\rangle,
\end{aligned}
$$

where $g i$ and $f j$ are the initial and final vibronic states, respectively, and $\phi(Q)$ represents the vibrational functions. $M_{g f}\left(Q_{0}\right)$ is the electronic transition dipole moment function,

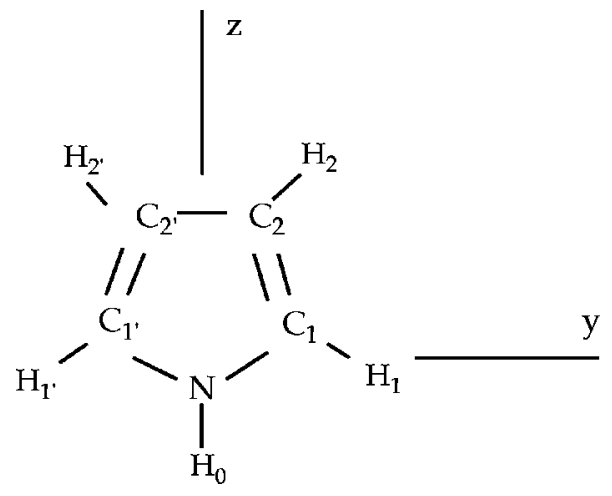

FIG. 1. Molecular structure, labeling, and orientation for pyrrole.

evaluated at the initial state geometry by means of the CASSI method. ${ }^{33}$ The vibrational wave functions use force fields taken directly from the second derivatives, with no adjustment for anharmonicities. The Condon approximation requires the neglect of all but the first term, which usually dominates the one-photon symmetry allowed transitions. The other terms represent an electronic transition moment induced by distortion away from the equilibrium. This is known as Herzberg-Teller vibronic coupling and the present approach will include first derivatives only, as shown in the formula above. The computed spectra include excitations up to four quanta in the excited state. A full explanation of the procedure followed to obtain the vibronic intensities can be found elsewhere. ${ }^{34,36}$ In order to account for the finite experimental resolution and for the degrees of freedom not considered here (such as rotation), the vibrational spectra computed in the present study were convoluted with a Lorentzian function of full width at half maximum (FWHM) corresponding to a lifetime of $33 \mathrm{fs}$. The temperature was considered by including a population of the vibrational states through a Boltzmann distribution at $10 \mathrm{~K}$, therefore the presence of intense hot bands in the computed spectrum can be ruled out.

All calculations were carried out using the MOLCAS-5 quantum-chemical package. ${ }^{37}$

\section{RESULTS AND DISCUSSION}

\section{A. The lowest-energy absorption band of pyrrole}

Figure 1 displays the structure and labeling of the pyrrole molecule. Table I compiles the main equilibrium bond distances for the ground and low-lying excited states of the molecule. The determined parameters for the ground state are in agreement with the structure characterized experimentally from microwave spectra of isotopic pyrroles. ${ }^{38}$ The geometry optimizations, performed numerically at the CASPT2 level for each of the states, were restricted to the planar $C_{2 v}$ symmetry. As shall be discussed below, the computed CASPT2 force fields confirm the planar, $\mathrm{C}_{2 v}$, character of the ground and four Rydberg states. Imaginary frequencies found for some of the out-of-plane modes in the two optimized geometries of the valence excited states indicate that they may have a nonplanar optimal conformations. Geometry optimizations performed also numerically at the CASPT2 level by using the $6-31 \mathrm{G}^{*}$ basis set and without 
TABLE I. Equilibrium geometries for the ground and low-lying singlet excited states of neutral pyrrole and the ground state of the positive ion optimized at the CASPT2 level employing the $\mathrm{ANO}^{\mathrm{a}}$ $\mathrm{C}, \mathrm{N}[4 s 3 p 2 d] / \mathrm{H}[3 s 2 p]+1 s 1 p$ basis set.

\begin{tabular}{lcccccccc}
\hline \hline Parameter $^{\mathrm{b}}$ & $1{ }^{1} A_{1}{ }^{\mathrm{c}}$ & ${ }^{1} A_{1}\left(\pi \pi^{*}\right)$ & ${ }^{1} B_{2}\left(\pi \pi^{*}\right)$ & ${ }^{1} A_{2}(3 s)$ & ${ }^{1} A_{2}(3 p)$ & ${ }^{1} B_{1}(3 p)$ & ${ }^{1} B_{2}(3 p)$ & $1{ }^{2} A_{2}$ \\
\hline$r\left(\mathrm{~N}-\mathrm{C}_{1}\right)$ & $1.376(1.370)$ & 1.396 & 1.438 & 1.351 & 1.365 & 1.363 & 1.359 & 1.357 \\
$r\left(\mathrm{C}_{1}-\mathrm{C}_{2}\right)$ & $1.386(1.382)$ & 1.455 & 1.450 & 1.440 & 1.433 & 1.433 & 1.436 & 1.427 \\
$r\left(\mathrm{C}_{2}-\mathrm{C}_{2}\right)$ & $1.422(1.417)^{\mathrm{d}}$ & 1.473 & 1.385 & 1.375 & 1.378 & 1.380 & 1.374 & 1.380 \\
$r\left(\mathrm{~N}-\mathrm{H}_{0}\right)$ & $1.003(0.996)$ & 1.004 & 1.001 & 1.058 & 1.015 & 1.009 & 1.008 & 1.012 \\
$r\left(\mathrm{C}_{1}-\mathrm{H}_{1}\right)$ & $1.075(1.076)$ & 1.076 & 1.077 & 1.080 & 1.079 & 1.078 & 1.077 & 1.078 \\
$r\left(\mathrm{C}_{2}-\mathrm{H}_{2}\right)$ & $1.076(1.077)$ & 1.075 & 1.076 & 1.076 & 1.074 & 1.076 & 1.076 & 1.074 \\
\hline \hline
\end{tabular}

${ }^{\mathrm{a}}$ See Ref. 30.

${ }^{\mathrm{b}}$ Bond distances in $\AA$.

${ }^{\mathrm{c}}$ Experimental data within parentheses taken from microwave spectra. See Ref. 38.

${ }^{\mathrm{d}}$ From the angles reported in the microwave spectra (see Ref. 38) the $r\left(\mathrm{C}_{2}-\mathrm{C}_{2},\right)$ bond length yields $1.415 \AA$.

symmetry constraints ${ }^{39}$ obtained a planar $\mathrm{C}_{2 v}$ geometry for the ${ }^{1} B_{2}$ valence state and a nonplanar structure for the $2{ }^{1} A_{1}$ valence state. Deviation from planarity of the ${ }^{1} B_{2}$ valence excited state, if any, should be rather small.

Upon electronic excitation the six excited states have in common the increase of the $\mathrm{C}_{1}-\mathrm{C}_{2}$ bond distance. For the valence excited states this can be easily rationalized taking into account the bonding interaction between the $\mathrm{C}_{1}$ and $\mathrm{C}_{2}$ atoms in the valence $1 a_{2}$ highest occupied molecular orbital (HOMO) and its antibonding nature in the valence virtual MOs (both $3 b_{1}$ and $2 a_{2}$ ). The increase of the $\mathrm{C}_{1}-\mathrm{C}_{2}$ bond length with respect to the ground state is not so pronounced for the Rydberg states because they merely involve a oneelectron promotion from the HOMO to a $3 s, 3 p$ Rydberg-like MO. As expected, the optimized geometry for the computed Rydberg states closely resembles the ground state geometry of the cation $\left(1^{2} A_{2}\right.$ state). Similar reasoning can be applied to explain the decrease obtained in the $\mathrm{C}_{2}-\mathrm{C}_{2}$, bond distance of the ${ }^{1} B_{2}\left(\pi \pi^{*}\right)$ valence state, involving primarily the HOMO and lowest unoccupied molecular orbital (LUMO) with antibonding $\left(1 a_{2}\right)$ bonding $\left(3 b_{1}\right)$ character between the $\mathrm{C}_{2}$ and $\mathrm{C}_{2}$, atoms, respectively. Also, the $\mathrm{N}-\mathrm{C}_{1}$ distance increases for the ${ }^{1} B_{2}\left(\pi \pi^{*}\right)$ valence state, because the HOMO and LUMO orbitals, with regard to this bond, are nonbonding and antibonding, respectively. The obtained CASPT2 geometries are similar to those computed at the coupled-cluster singles doubles (CCSD) level ${ }^{12}$ for the ground and the three $3 p$ Rydberg states. The maximum differences are found in the $\mathrm{C}_{1}-\mathrm{C}_{2}$ length, 0.002 and $0.013 \AA$ shorter in the ground and $3 p$ Rydberg states, respectively, at the CASPT2 level of calculation.

The adiabatic band origins $(0-0), T_{e}$, and vertical excitation energies $\left(T_{v}\right)$ are listed in Table II. The difference between $T_{v}$ and $T_{e}$ energies is at most $0.2 \mathrm{eV}$ and can be related to the structural change of the corresponding excited state. On the other hand, the difference between the $0-0$ and $T_{e}$ energies accounts for the zero point energy (ZPE) correction and is small $(<0.1 \mathrm{eV})$ in all cases. Before entering into a discussion of the vibrational profiles, several conclusions can be made. First, as mentioned in Sec. I, we notice the clear one-photon forbidden character of the very weak transition observed starting at $4.96 \mathrm{eV}$, already suggested by Bavia et al. ${ }^{16}$ as the origin of the $\mathrm{HOMO} \rightarrow 3 s{ }^{1} A_{2}$ transition. Our computed band origin at $4.98 \mathrm{eV}$ and vertical excitation at $5.22 \mathrm{eV}$ fully agree with both the recorded band origin $(4.96 \mathrm{eV})$ and the observed maximum $(5.22 \mathrm{eV})$ for the $1 a_{2} \rightarrow 3 s$ electronic transition. ${ }^{16}$ We believe that the discussion on the band assignment ${ }^{13,15,17,40}$ has reached its end. Second, the computed results confirm that both vertically and adiabatically the $2{ }^{1} A_{1}\left(\pi \pi^{*}\right)$ excited state lies below the $1{ }^{1} B_{2}\left(\pi \pi^{*}\right)$ excited state. The two states are found to be involved in the lowest-energy absorption band, although the intensity of the transition to the $2{ }^{1} A_{1}\left(\pi \pi^{*}\right)$ state is predicted to be much lower. Third, the $1{ }^{1} B_{2}\left(\pi \pi^{*}\right)$ excited state is calculated to be below the states of the HOMO $\rightarrow 3 p$ Rydberg series. Although there is a certain degree of uncertainty in the position of the ${ }^{1} B_{2}$ states, probably larger than that of the other states, it should not significantly affect the final conclusions.

The discussion of the remaining assignments requires a more detailed analysis of the vibrational structure of the absorption bands. Table III compiles the CASPT2 (unless indicated) harmonic frequencies corresponding to the seven optimized states of pyrrole. Figure 2 reproduces the measured optical spectrum of pyrrole in the vapor ${ }^{15}$ together with our computed spectra, which includes the summed intensity for the absorption transitions to the six mentioned excited states. The agreement between the experimental and the theoretical profiles is noticeable. As reported in Table II, most of the intensity in the computed band originates in the transition to the $1^{1} B_{2}\left(\pi \pi^{*}\right)$ valence excited state, with an oscillator

TABLE II. Computed excitation energies $(\mathrm{eV})$ and oscillator strengths $(f)$ together with available experimental data.

\begin{tabular}{lccccc}
\hline \hline \multicolumn{1}{c}{ State } & $0-0$ & $T_{e}$ & Vertical & $\operatorname{Exp}(0-0)$ & $f^{\mathrm{a}}$ \\
\hline $1{ }^{1} A_{2}(3 s)$ & 4.98 & 5.02 & 5.22 & $4.96^{\mathrm{b}}$ & forbidden \\
$2{ }^{1} A_{1}\left(\pi \pi^{*}\right)$ & 5.57 & 5.67 & 5.82 & & 0.036 \\
$1{ }^{1} B_{2}\left(\pi \pi^{*}\right)$ & 5.71 & 5.73 & 5.87 & $5.70^{\mathrm{c}, \mathrm{d}}$ & 0.209 \\
$2{ }^{1} A_{2}\left(3 p_{z}\right)$ & 5.77 & 5.83 & 5.97 & & forbidden \\
$1{ }^{1} B_{1}\left(3 p_{y}\right)$ & 5.90 & 5.87 & 5.87 & $5.86^{\mathrm{d}}$ & 0.026 \\
$2{ }^{1} B_{2}\left(3 p_{x}\right)$ & 5.91 & 5.93 & 6.09 & $5.70^{\mathrm{d}}$ & 0.013 \\
\hline \hline
\end{tabular}

${ }^{a}$ Computed at the ground state geometry (PMCAS-CI TDMs and MSCASPT2 energies).

${ }^{\mathrm{b}}$ Estimated band origin and assignment from the optical spectrum (see Ref. 16).

${ }^{\mathrm{c}}$ Preferred assignment based on the present results and the analysis of Bavia et al. (Ref. 16).

${ }^{\mathrm{d}}$ From optical and EEL spectra by Palmer et al. (Ref. 11). 
TABLE III. Computed vibrational harmonic frequencies $\left(\mathrm{cm}^{-1}\right)$ for the low-lying excited states of pyrrole at the CASPT2 level of calculation.

\begin{tabular}{ccccccccc}
\hline \hline Symmetry & Mode $^{\mathrm{a}}$ & $1{ }^{1} A_{1}{ }^{\mathrm{b}}$ & ${ }^{1} A_{2}(3 s)$ & ${ }^{1} A_{1}\left(\pi \pi^{*}\right)^{\mathrm{c}}$ & ${ }^{1} B_{2}\left(\pi \pi^{*}\right)$ & ${ }^{1} A_{2}(3 p)$ & ${ }^{1} B_{1}(3 p)$ & ${ }^{1} B_{2}(3 p)$ \\
\hline$a_{1}$ & $\nu_{1}\left(\nu_{9}\right)$ & $3600(3531)$ & 3327 & 3724 & 3594 & 3581 & 3545 & 3463 \\
& $\nu_{2}\left(\nu_{8}\right)$ & $3377(3145)$ & 3292 & 3361 & 3333 & 3255 & 3327 & 3384 \\
& $\nu_{3}\left(\nu_{7}\right)$ & $3329(3129)$ & 3245 & 3333 & 3312 & 3188 & 3293 & 3370 \\
& $\nu_{4}\left(\nu_{6}\right)$ & $1480(1467)$ & 1570 & 1610 & 1545 & 1560 & 1566 & 1627 \\
& $\nu_{5}\left(\nu_{5}\right)$ & $1461(1382)$ & 1461 & 1517 & 1385 & 1405 & 1466 & 1467 \\
& $\nu_{6}\left(\nu_{4}\right)$ & $1174(1144)$ & 1141 & 1185 & 1132 & 1185 & 1164 & 1164 \\
& $\nu_{7}\left(\nu_{3}\right)$ & $1075(1074)$ & 1051 & 1082 & 1056 & 932 & 1114 & 1113 \\
& $\nu_{8}\left(\nu_{2}\right)$ & $1031(1016)$ & 1023 & 1032 & 973 & 900 & 932 & 998 \\
& $\nu_{9}\left(\nu_{1}\right)$ & $904(881)$ & 854 & 813 & 725 & 532 & 897 & 910 \\
$a_{2}$ & $\nu_{10}\left(\nu_{20}\right)$ & $860(869)$ & 910 & 311 & 820 & 940 & 905 & 890 \\
& $\nu_{11}\left(\nu_{19}\right)$ & $661(710)$ & 862 & $238 \mathrm{i}$ & 592 & 846 & 734 & 658 \\
& $\nu_{12}\left(\nu_{18}\right)$ & $610(618)$ & 491 & $607 i$ & 383 & 501 & 451 & 455 \\
$b_{2}$ & $\nu_{13}\left(\nu_{17}\right)$ & $3309(3145)$ & 3282 & 3356 & 3299 & 3342 & 3305 & 3305 \\
& $\nu_{14}\left(\nu_{16}\right)$ & $3275(3129)$ & 3263 & 3319 & 3286 & 3324 & 3229 & 3292 \\
& $\nu_{15}\left(\nu_{15}\right)$ & $1560(1530)$ & 1442 & 1626 & 2277 & 1628 & 1530 & 1515 \\
& $\nu_{16}\left(\nu_{14}\right)$ & $1458(1422)$ & 1305 & 1510 & 1315 & 1450 & 1348 & 1335 \\
& $\nu_{17}\left(\nu_{13}\right)$ & $1313(1287)$ & 1285 & 1394 & 1207 & 1391 & 1162 & 1300 \\
& $\nu_{18}\left(\nu_{12}\right)$ & $1178(1134)$ & 1052 & 1237 & 1115 & 1067 & 949 & 1105 \\
& $\nu_{19}\left(\nu_{11}\right)$ & $1071(1048)$ & 988 & 1051 & 927 & 967 & 690 & 1006 \\
& $\nu_{20}\left(\nu_{10}\right)$ & $871(865)$ & 768 & 800 & 779 & 598 & 625 & 767 \\
$b_{1}$ & $\nu_{21}\left(\nu_{24}\right)$ & $811(826)$ & 891 & 497 & 622 & 898 & 969 & 825 \\
& $\nu_{22}\left(\nu_{23}\right)$ & $717(721)$ & 725 & 280 & 330 & 799 & 831 & 735 \\
& $\nu_{23}\left(\nu_{22}\right)$ & $633(601)$ & 629 & $111 \mathrm{i}$ & $143 \mathrm{i}$ & 582 & 818 & 601 \\
& $\nu_{24}\left(\nu_{21}\right)$ & $464(474)$ & 467 & $660 \mathrm{i}$ & $381 \mathrm{i}$ & 415 & 567 & 466 \\
\hline \hline
\end{tabular}

${ }^{a}$ Herzberg's convention (Ref. 41). Lord and Miller convention within parentheses (Ref. 44).

${ }^{\mathrm{b}}$ Ground state harmonic frequencies. Experimental fundamentals (Ref. 43) within parentheses.

${ }^{\mathrm{c}} \mathrm{CASSCF}$ force field at the CASPT2 optimized geometry.

strength at the ground state geometry of $0.209,1$ order of magnitude larger than for the other transitions. Figure 3 contains the individual contributions for each of the electronic transitions, where the relative extinction coefficients of some of the bands have been enhanced.

Most of the analyses of the pyrrole spectrum in the vapor have identified two clear vibrational progressions in the 5.5$6.2 \mathrm{eV}$ range of energies. Bavia et al. ${ }^{16}$ assigned the observed peak at $5.64 \mathrm{eV}$ to the origin of a transition which they suggested to correspond to the $1{ }^{1} B_{2}\left(\pi \pi^{*}\right)$ valence excited state, extended to higher frequencies beneath a set of Rydberg transitions. The peak was reported to be broader than the typical Rydberg transitions and not to be affected in a large temperature range (ruling out the possibility of being a hot band). The assignment was confirmed to be in agreement with the findings in the crystal and liquid spectra of pyrrole $^{16}$ where the presence of one or even two valence transitions seemed clear. Palmer et al. ${ }^{11}$ measured the peak at $5.70 \mathrm{eV}$ and tentatively assigned it to the origin of the transition to the ${ }^{1} B_{2}\left(3 p_{x}\right)$ Rydberg state. The band does not carry the photoelectron spectrum (PES) vibrational profile, a sign which would indicate its unequivocal Rydberg character. Palmer et $a l .{ }^{11}$ ascribed this fact to the mixing of the state with the underlying ${ }^{1} B_{2}\left(\pi \pi^{*}\right)$ valence state. A second band, and the most intense peak of the spectrum, measured at 5.86 $\mathrm{eV}$, has been clearly and unambiguously assigned to the origin of the $1 a_{2} \rightarrow 3 p_{y}$ Rydberg transition to the $1{ }^{1} B_{1}\left(3 p_{y}\right)$ state (notice the change in labels in the different papers because of the respective molecular orientations). The band carries the PES vibrational signature corresponding to the first ionization potential from the $1 a_{2}$ orbital $^{11,14}$ confirming the Rydberg nature of the transition. The three main experimental studies ${ }^{11,14,16}$ preferred the $1{ }^{1} B_{1}\left(3 p_{y}\right)$ assignment to the possibility of a transition to the ${ }^{1} B_{2}\left(3 p_{x}\right)$ Rydberg state, expected to interact strongly with the valence state and lead to broader bands. ${ }^{16}$ Other results obtained in a multiphoton ionization study of pyrrole ${ }^{18}$ are too uncertain for the $n=3$ Rydberg series to be of any use, especially when they disagree with all the other experimental evidences.

Our computed results confirm most of the described assignments and proposals while resolving some of the uncertainties. According to the energy order, the first of the transitions forming the 5.5-6.5 eV band of pyrrole corresponds to the $2{ }^{1} A_{1}\left(\pi \pi^{*}\right)$ valence excited state, having a band origin calculated at $5.57 \mathrm{eV}$ and a vertical energy of $5.82 \mathrm{eV}$. The vertical oscillator strength is 0.036 . Although the overall intensity is similar to that of the optically allowed computed Rydberg transitions, it is clearly spread over a larger number of vibronic transitions (see Fig. 3), unlike the Rydberg bands which have the bulk of their intensities concentrated in the $0-0$ band. Therefore, no prominent peak can be expected to arise from the $2{ }^{1} A_{1}\left(\pi \pi^{*}\right)$ transition. No evidence of any important peak has been found experimentally in the lowenergy tail of the band, which decreases in intensity until the observed weak shoulder near $5.2 \mathrm{eV}$ (Ref. 15) corresponding to the $1{ }^{1} A_{2}(3 s)$ state. Second in energy, the $1{ }^{1} B_{2}\left(\pi \pi^{*}\right)$ valence state is computed. The origin of its related transition is obtained at $5.71 \mathrm{eV}$ and its vertical energy and oscillator strength are $5.87 \mathrm{eV}$ and 0.209 , respectively. The transition to the $2{ }^{1} A_{2}\left(3 p_{z}\right)$ state is next in energy, at $5.77 \mathrm{eV}$. It is 

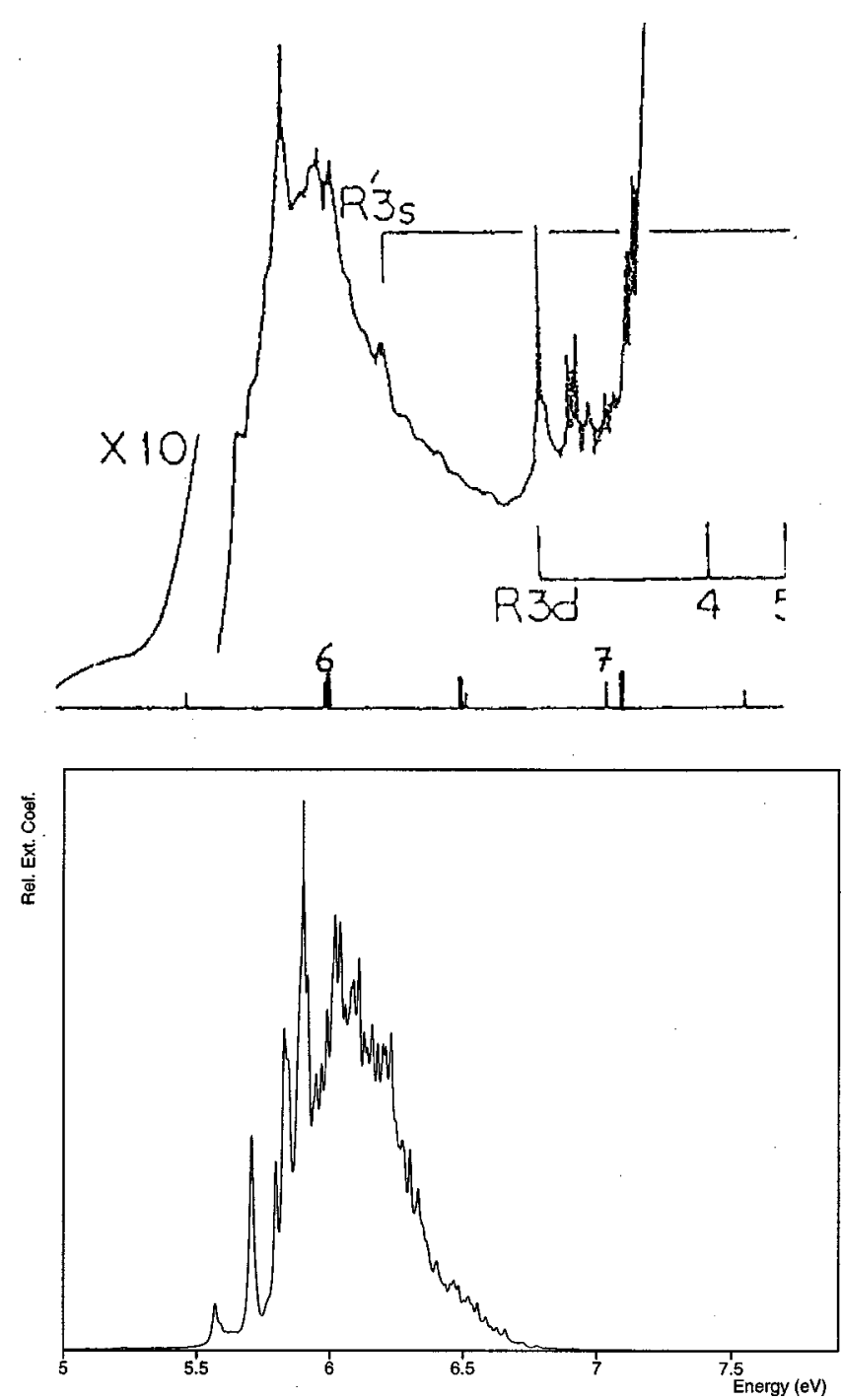

FIG. 2. Experimental (upper) (Ref. 15) and theoretical (lower) absorption low-energy band of the pyrrole molecule. The experimental band is obtained from the vapor optical spectrum (Ref. 15). The CASPT2/MS-CASPT2 contains the summed absorption bands from the ground to each of the six low-lying singlet excited states. Relative extinction coefficients in abcises.

optically forbidden and cannot be detected in the one-photon spectrum. The band origin of the transition to the $1{ }^{1} B_{1}\left(3 p_{y}\right)$ Rydberg state is found at $5.90 \mathrm{eV}$ with a vertical oscillator strength of 0.026 , while the origin of the Rydberg transition to the $2{ }^{1} B_{2}\left(3 p_{x}\right)$ state is computed at $5.91 \mathrm{eV}$ with a band oscillator strength of 0.013 .

In agreement with Bavia et al. ${ }^{16}$ we ascribe the $5.70 \mathrm{eV}$ band, also detected by Palmer et al., ${ }^{11}$ to the band origin of the valence $1{ }^{1} B_{2}\left(\pi \pi^{*}\right)$ band, computed at $5.71 \mathrm{eV}$ and clearly visible both in the summed spectrum of Fig. 2 and in the individual $1^{1} B_{2}\left(\pi \pi^{*}\right)$ spectrum of Fig. 3 as the $0-0$ transition. No other transitions are visible in the vicinity of this band and the Rydberg transition origins are $0.2 \mathrm{eV}$ shifted to higher energies. The assignment is not in disagreement with Palmer et al., ${ }^{11}$ who suggested the correspondence to the Rydberg ${ }^{1} B_{2}$ state to fill its apparent absence in the spectrum, despite contradictory evidence as to the lack of a PES signature. The current results are consistent with the available experimental data and help to rationalize the re- corded profiles. We have, however, to recall that the position of the valence and Rydberg ${ }^{1} B_{2}$ states is computed within 0.2 $\mathrm{eV}$, that is, the expected error bar of the employed method.

The most intense band in our spectrum is computed at $5.90 \mathrm{eV}$ (see Fig. 2). It is formed mainly by the sum of intensities of three transitions: $1{ }^{1} B_{2}\left(\pi \pi^{*}\right), \quad{ }^{1} B_{1}\left(3 p_{y}\right)$, and $2{ }^{1} B_{2}\left(3 p_{x}\right)$. The largest contribution originates obviously from the intense $1{ }^{1} B_{2}\left(\pi \pi^{*}\right)$ intravalence transition, however, as most of the intensity of the $1{ }^{1} B_{1}\left(3 p_{y}\right)$ band is carried by its $0-0$ transition, the added strengths combining to produce a sharp peak. We therefore confirm the previous assignment ${ }^{11,14,16}$ of the most intense $5.86 \mathrm{eV}$ band basically to the transition to the $1{ }^{1} B_{1}\left(3 p_{y}\right)$ Rydberg state, with an underlying contribution of the $1^{1} B_{2}\left(\pi \pi^{*}\right)$ valence transition. As the computed band origin for the $2{ }^{1} B_{2}\left(3 p_{x}\right)$ band is close to this energy, $5.91 \mathrm{eV}$, there will be also a participation of this transition to the overall intensity, although its vertical oscillator strength is computed lower than those of the other states, 0.013. As the accuracy of our calculations is not expected to be below $0.05 \mathrm{eV}$, it is not possible to predict that the observed $5.86 \mathrm{eV}$ peak also has a contribution of the $2{ }^{1} B_{2}\left(3 p_{x}\right)$ transition. We prefer the classical assignment of the band to the $1{ }^{1} B_{1}\left(3 p_{y}\right)$ transition and simply suggest that the weaker $2{ }^{1} B_{2}\left(3 p_{x}\right)$ band cannot be directly observed because it lies beneath the more intense $1{ }^{1} B_{2}\left(\pi \pi^{*}\right)$ and $1{ }^{1} B_{1}\left(3 p_{y}\right)$ bands.

A comparison of the present results with the other theoretical data reported recently ${ }^{9-12}$ indicates that similar conclusions and assignments are obtained for the $3 s$ and $3 p$ Rydberg states. We restrict the comparison to the most complete results at the $\mathrm{CC}$ level estimated to be the best, CCSDR(3) plus basis set correction effects. ${ }^{12}$ They include vertical and $0-0$ transitions for the Rydberg $3 s, 3 p$ states. The vertical excitation energies $(\mathrm{eV})$ at the CASPT2 (CC) levels for the ${ }^{1} A_{2}(3 s), \quad 2^{1} A_{2}\left(3 p_{z}\right), \quad 1^{1} B_{1}\left(3 p_{y}\right)$, and $2{ }^{1} B_{2}\left(3 p_{x}\right)$ states are: 5.22 (5.20), 5.97 (5.94), 5.87 (5.95), and $6.09(6.04) \mathrm{eV}$, respectively. The maximum difference in energy is $0.08 \mathrm{eV}$ for the $1{ }^{1} B_{1}\left(3 p_{y}\right)$ state. Regarding the 0-0 transitions, both including the ZPE correction, the corresponding results are: 4.98 (4.83), 5.77 (5.75), 5.90 (5.84), and $5.91(5.80) \mathrm{eV}$, respectively in the same order as before. The agreement of the two sets of values is good in both the vertical and adiabatic Rydberg transitions. The larger discrepancy is observed for the $2{ }^{1} B_{2}\left(3 p_{x}\right)$ 0-0 transition, which is $0.11 \mathrm{eV}$ lower at the $\mathrm{CC}$ level of calculation. Also for the $2{ }^{1} B_{2}\left(3 p_{x}\right)$ transition we find the largest discrepancy between the CC and PMCAS-CI/MS-CASPT2 vertical oscillator strengths, five times larger in the CC calculations. These differences are better reflected in the results for the valence states, where only vertical CC excitation energies are available. The vertical absorptions at the MS-CASPT2 level have been computed at 5.82 and $5.87 \mathrm{eV}$, respectively, for the $2{ }^{1} A_{1}\left(\pi \pi^{*}\right)$ and $1{ }^{1} B_{2}\left(\pi \pi^{*}\right)$ valence states, with oscillator strengths 0.036 and 0.209 , respectively. The $\mathrm{CC}$ values are $6.37(0.001)$ and $6.57 \mathrm{eV}(0.033)$, respectively, with the oscillator strengths within parentheses. The discrepancy in the $2{ }^{1} A_{1}\left(\pi \pi^{*}\right)$ excitation energy can be understood because of the problems of the $\mathrm{CC}$ methods on dealing with states with large multiconfigurational character. In this case also there 

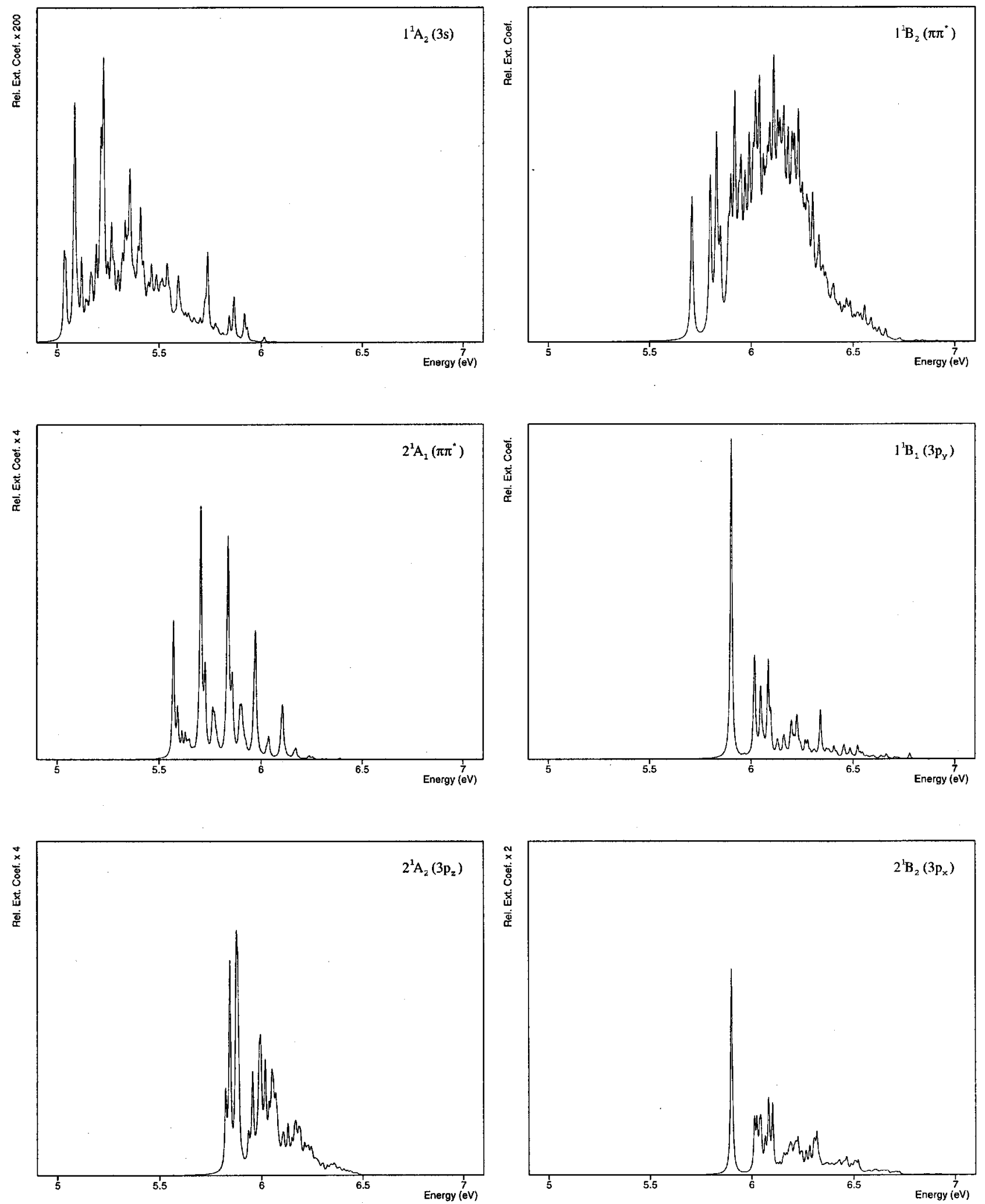

FIG. 3. Computed absorption bands from the ground to each of the six low-lying singlet excited states of pyrrole. Relative extinction coefficients in abcises. Some of the intensity scales have been enhanced.

are large contributions ( $>25 \%)$ of doubly excited configurations. Other multireference treatments such and MRMP placed the state near the MS-CASPT2 results, at $6.01 \mathrm{eV}^{9}{ }^{9}$ The discrepancy in the vertical excitation energy of the $1{ }^{1} B_{2}\left(\pi \pi^{*}\right)$ valence state is even larger and it will be discussed in the next sections.

\section{B. The vibrational structure of the low-lying electronic bands of pyrrole}

Table IV compiles the computed and experimental excitation energies and energy differences, oscillator strengths, and proposed assignments for the vibrational bands of the 
TABLE IV. Computed and experimental excitation energies and energy differences $(E / \mathrm{eV})$, oscillator strengths $(f)$, and assigments for the vibrational bands of the low-lying electronic states of pyrrole.

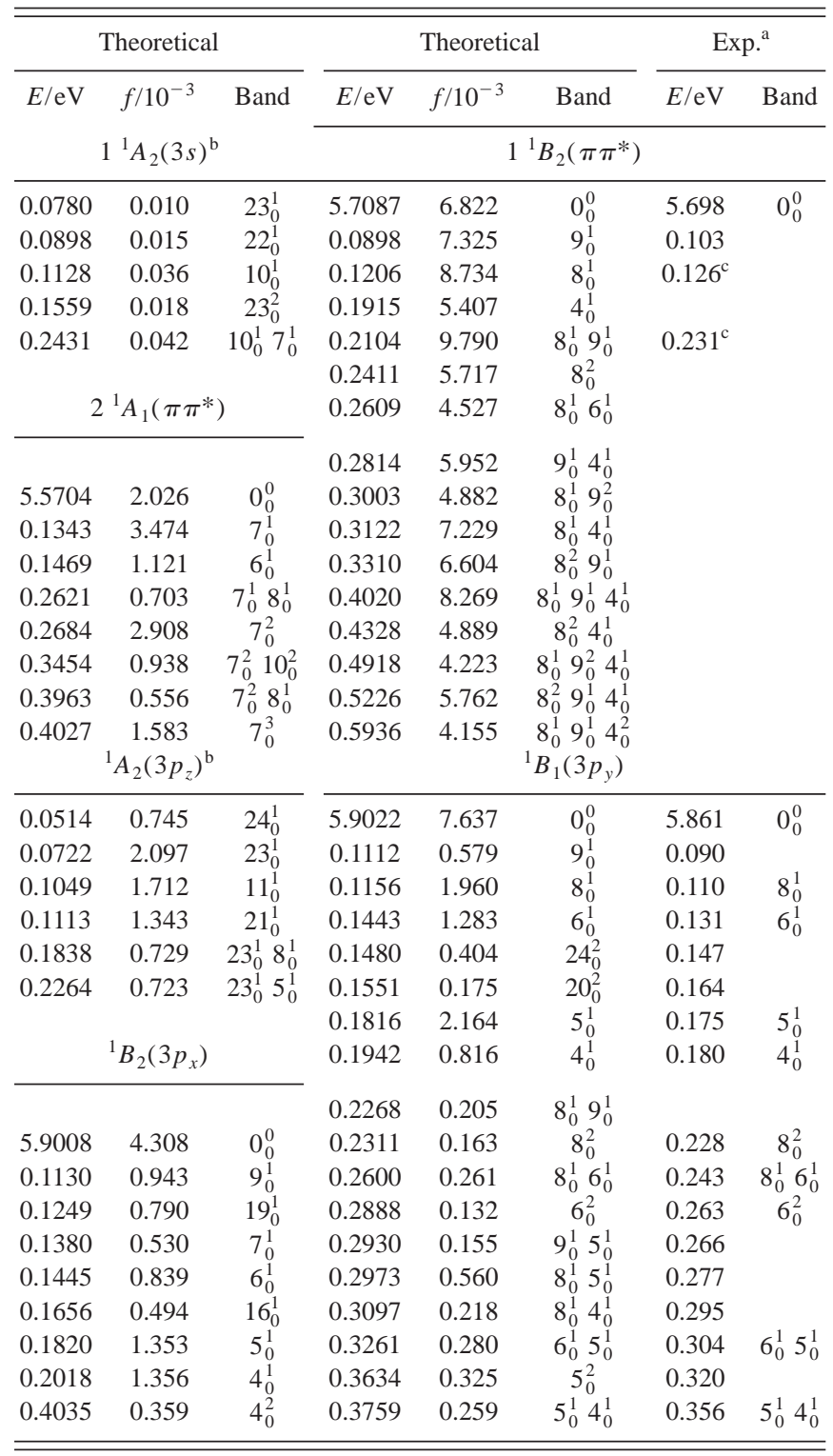

${ }^{a}$ Energies taken from Palmer et al. (Ref. 11) similar to those in Derrick et al. (Ref. 14) and Bavia et al. (Ref. 16).

${ }^{\mathrm{b}}$ The forbidden $0_{0}^{0}$ bands are placed at $4.9784 \mathrm{eV}\left({ }^{1} A_{2}(3 s)\right)$ and $5.7222 \mathrm{eV}$ $\left({ }^{1} A_{2}\left(3 p_{z}\right)\right)$.

${ }^{\mathrm{c}}$ Observed at 5.818 and $5.923 \mathrm{eV}$, respectively, by Bavia et al. (Ref. 16).

low-lying singlet excited states of pyrrole. Notice that the bands drawn in Figs. 2 and 3 correspond to the sum of the oscillator strengths obtained within the present model once a FWHM has been selected and therefore they have to be considered as relative extinction coefficients. The directly computed oscillator strengths for the vibronic bands are those listed in Table IV, while the total area forming the absorption band corresponds to the total computed oscillator strength.

There are three basic sources of information on the vibrational structure of the bands of pyrrole: the analyses of the optical spectra by Derrick et al. ${ }^{14}$ Bavia et al. ${ }^{16}$ and the most recent and more extended study reported by Palmer et al. ${ }^{11}$ The energies in Table IV have been taken from the latter, although the three studies agree within less than 5
meV. The normal modes have been labeled according to Herzberg's notation. ${ }^{41}$ There is a general agreement on the assignment of most of the vibrational structure found between 5.8 and $6.2 \mathrm{eV}$ to the $1^{1} B_{1}\left(3 p_{y}\right)$ transition, with its band origin at $5.86 \mathrm{eV}$ (computed $5.90 \mathrm{eV}$ ). At lower energies several transitions were reported and assigned either to the $1{ }^{1} B_{2}\left(\pi \pi^{*}\right)$ valence transition ${ }^{16}$ or to the $2{ }^{1} B_{2}\left(3 p_{x}\right)$ Rydberg excitation. ${ }^{11}$

Starting with the low intensity bands, $1{ }^{1} A_{2}(3 s)$ extends from its one-photon forbidden origin at $4.98 \mathrm{eV}$ in a somewhat long progression up to $6.0 \mathrm{eV}$. The band will be visible only in the low-energy region where no other transition is present. Notice that the intensity for this band in Fig. 3 is enhanced 200 times. The most intense peaks correspond to out-of-plane vibrations, in particular excitation $10_{0}^{1}\left(a_{2}\right)$ in which the hydrogens $\mathrm{H}_{2}$ and $\mathrm{H}_{2}^{\prime}$ break the planarity. A conventional nomenclature has been introduced ${ }^{42}$ where the mode is represented by its number and the number of quanta in the lower or upper state as a subscript or a superscript, respectively. The out-of-plane $a_{2}$ and $b_{1}$ modes act as coupling modes for the in-plane vibrations, such in the $10_{0}^{1} 7_{0}^{1}$ band. The overall profile of the $1{ }^{1} A_{2}(3 s)$ band has a computed maximum at $5.22 \mathrm{eV}$ corresponding to the observed intensity maximum near $5.2 \mathrm{eV} \cdot{ }^{15}$ The $2{ }^{1} A_{1}\left(\pi \pi^{*}\right)$ band is next in energy and it also has low intensity. The computed summed spectrum in Fig. 2 has a set of bands at $5.6 \mathrm{eV}$ related to the $2{ }^{1} A_{1}\left(\pi \pi^{*}\right)$ valence transition. These peaks are not observed in the experimental spectrum. The energy of the transition origin has probably been underestimated at the MS-CASPT2 level or the calculated oscillator strength is too large. The corresponding vibrational progression is also large because of the changes in the length of the $\mathrm{C}-\mathrm{C}$ bonds with respect to the ground state values. The most intense progression seats on mode $\nu_{7}$, corresponding to the plus combination of the symmetric stretchings $\mathrm{C}_{1}-\mathrm{C}_{2}\left(\mathrm{C}_{1}^{\prime}-\mathrm{C}_{2}^{\prime}\right)$ and $\mathrm{N}-\mathrm{C}_{1}\left(\mathrm{~N}-\mathrm{C}_{1}^{\prime}\right)$. Next in energy is the $2{ }^{1} A_{2}\left(3 p_{z}\right)$ band, also a one-photon forbidden transition. The intensity of the band is not as low as might have been expected. Because of its forbidden character all the intensity is obtained from the first-order elements in the Herzberg-Teller expansion, in which the $b_{1}, a_{2}$, and $b_{2}$ vibrations act as coupling modes for the totally symmetric vibrations. In particular, the most intense progressions correspond to mode $\nu_{23}$, involving outof-plane displacements in all hydrogens. The band is located in the vicinity of both $2{ }^{1} A_{1}\left(\pi \pi^{*}\right)$ and $1{ }^{1} B_{2}\left(\pi \pi^{*}\right)$ valence bands, from which the transition will borrow most of the intensity through the coupling with $a_{2}$ and $b_{1}$ modes, respectively.

The absorption band to the $1{ }^{1} B_{2}\left(\pi \pi^{*}\right)$ valence state is intense and displays a large number of vibrational progressions. Our computed band origin at $5.709 \mathrm{eV}$ can be tentatively assigned to the clear peak observed at $5.698 \mathrm{eV}$, related to the $1{ }^{1} B_{2}\left(\pi \pi^{*}\right)$ valence transition by Bavia et al. ${ }^{16}$ and ${ }^{1} B_{2}\left(3 p_{x}\right)$ Rydberg transition by Palmer et al. ${ }^{11}$ In Table IV we have ascribed three observed, but unassigned, peaks to the most intense bands computed in our spectrum. Bands labeled as $9_{0}^{1}$ and $8_{0}^{1}$ are clearly visible in the spectrum below the $1{ }^{1} B_{1}\left(3 p_{y}\right)$ origin and should correspond to a lower electronic transition. The peak assigned to the $8_{0}^{1} 9_{0}^{1}$ vibra- 
tional excitation of $1{ }^{1} B_{2}\left(\pi \pi^{*}\right)$ is too close to the $1^{1} B_{1}\left(3 p_{y}\right)$ band origin and the present assignment should be preferred. The most intense progressions in the absorption band to the $1{ }^{1} B_{2}\left(\pi \pi^{*}\right)$ valence state correspond to excitations to symmetric $a_{1}$ modes, in particular modes $\nu_{4}, \nu_{8}$, and $\nu_{9}$, composed mainly by the bending of $\mathrm{C}_{1}-\mathrm{C}_{2}-\mathrm{H}_{2}$, stretching of $\mathrm{N}-\mathrm{C}_{1}$, and bending of $\mathrm{N}-\mathrm{C}_{1}-\mathrm{C}_{2}$, respectively. Most of the observed and assigned vibrational bands in the spectrum of pyrrole in this energy range have been ascribed, however, to the $1{ }^{1} B_{1}\left(3 p_{y}\right)$ Rydberg transition. The agreement between our computed and the observed results strongly confirm these assignments. It is not surprising that a somewhat weak Rydberg transition prevails in a region where a much more intense valence state is placed. The intense transitions to valence states, because of their smaller lifetimes, are usually broader than those corresponding to Rydberg transitions, which are much sharper and clearly identified in the vapor. It is therefore common that the vibrational structure of a Rydberg band is identified, whereas the intense valence transition lies beneath as a broad band. ${ }^{15}$

The band origin of the $1{ }^{1} B_{1}\left(3 p_{y}\right)$ transition is observed as the highest peak at $5.861 \mathrm{eV}{ }^{11}$ Our result places the band at $5.902 \mathrm{eV}$ also with the largest intensity (see Fig. 3). Four $a_{1}$ modes are the main responsiblity of the measured intense progressions: $\nu_{5}, \nu_{8}, \nu_{6}$, and $\nu_{4}$ in order of decreasing intensity of their fundamental excitation. The main contributions to the normal modes can be described as the bending $\mathrm{N}-\mathrm{C}_{1}-\mathrm{H}_{1}$ plus stretching $\mathrm{C}_{1}-\mathrm{C}_{2}\left(\nu_{5}\right)$, bending $\mathrm{N}-\mathrm{C}_{1}-\mathrm{C}_{2}$ plus $\mathrm{C}_{1}-\mathrm{C}_{2}-\mathrm{H}_{2}\left(\nu_{8}\right)$, stretching $\mathrm{N}-\mathrm{C}_{1}$ plus bending $\mathrm{N}-\mathrm{C}_{1}-\mathrm{H}_{1}\left(\nu_{6}\right)$, and bending $\mathrm{N}-\mathrm{C}_{1}-\mathrm{H}_{1}$ plus $\mathrm{C}_{1}-\mathrm{C}_{2}-\mathrm{H}_{2}$ $\left(\nu_{4}\right)$ (obviously the symmetric atoms contribute equally). Apart from these main fundamentals, the remaining intensity is distributed basically among the overtones and combination bands built on these origins. The correspondence of the computed bands and assignments with the observed features is good in all cases. Compiled in Table IV, the so far unassigned bands are ascribed on the basis of the computed energy differences and band intensities. There are certain discrepancies in the labeling of the transitions worth mentioning. Bavia et al. ${ }^{16}$ assigned as $7_{0}^{1}$ the fundamental transition observed at $0.1308 \mathrm{eV}\left(1055 \mathrm{~cm}^{-1}\right)$ that we assign as $6_{0}^{1}$. They made the assignment on the basis of the ground states frequencies, where the fundamental vibration $\nu_{7}$ has the closest energy, $0.1332 \mathrm{eV}\left(1074 \mathrm{~cm}^{-1}\right) .{ }^{43}$ In the $1^{1} B_{1}\left(3 p_{y}\right)$ state $\nu_{7}$ increases by $40 \mathrm{~cm}^{-1}$ and comes close to $\nu_{6}$. Our computed intensity for the fundamental $7{ }_{0}^{1}$, composed almost exclusively by the minus combination of stretchings $\mathrm{N}-\mathrm{C}_{1}$ and $\mathrm{C}_{1}-\mathrm{C}_{2}$, is negligible. Therefore, in agreement with other authors ${ }^{11,14}$ we prefer the assignment to the $6_{0}^{1}$ fundamental. Something similar occurs for the $8_{0}^{1}$ fundamental, which is assigned by Derrick et al. ${ }^{14}$ and Palmer et $a l .{ }^{11}$ to mode $\nu_{1}\left(\mathrm{a}_{1}\right)$. They were following Lord and Miller's nomenclature, ${ }^{44}$ which begins the numbering of the frequencies by the lower, not the higher energy mode as in the present Herzberg's labeling. ${ }^{41}$ The authors ${ }^{11,14}$ selected label $\nu_{1}$ for the excitation at $0.110 \mathrm{eV}\left(877 \mathrm{~cm}^{-1}\right)$, corresponding to the lowest frequency $a_{1}$ mode in the ground state $\left(881 \mathrm{~cm}^{-1}\right)$. The situation differs in the $1{ }^{1} B_{1}\left(3 p_{y}\right)$ state, where the frequency of the second mode decreases almost
TABLE V. Computed excitation energies $(\mathrm{eV})$ and oscillator strengths $(f)$ for the two low-lying ${ }^{1} B_{2}$ states of pyrrole at different levels of calculation. ${ }^{a}$

\begin{tabular}{|c|c|c|c|c|c|c|c|c|}
\hline \multirow[b]{2}{*}{ State } & PT2 & $f$ & MPT2 & $f$ & PT2 & $f$ & MPT2 & $f$ \\
\hline & \multicolumn{4}{|c|}{ basis $A^{\mathrm{c}}$} & \multicolumn{4}{|c|}{ basis $B^{\mathrm{c}}$} \\
\hline \multicolumn{9}{|c|}{ Experimental geometry ${ }^{\mathrm{b}}$} \\
\hline${ }^{1} B_{2}(3 p)$ & $5.78^{\mathrm{d}}$ & $0.040^{\mathrm{d}}$ & 6.10 & 0.004 & 5.86 & 0.024 & 6.06 & 0.025 \\
\hline${ }^{1} B_{2}\left(\pi \pi^{*}\right)$ & $6.00^{\mathrm{d}}$ & $0.125^{\mathrm{d}}$ & 5.75 & 0.158 & 6.01 & 0.078 & 5.87 & 0.215 \\
\hline \multicolumn{9}{|c|}{ Optimized geometry ${ }^{\mathrm{b}}$} \\
\hline${ }^{1} B_{2}(3 p)$ & 5.81 & 0.064 & 6.17 & 0.102 & 6.00 & 0.073 & 6.09 & 0.013 \\
\hline${ }^{1} B_{2}\left(\pi \pi^{*}\right)$ & 6.88 & 0.171 & 5.86 & 0.049 & 6.08 & 0.057 & 5.87 & 0.209 \\
\hline
\end{tabular}

aPT2: CASPT2; MPT2: MS-CASPT2.

${ }^{b}$ Experimental (Ref. 38) and CASPT2 optimized (this work) ground state geometries.

${ }^{\mathrm{c}}$ Basis $A$ : ANO $4 s 3 p 1 d / 2 s 1 p+2 s 2 p 2 d$ (Ref. 7). Basis $B$ : ANO $4 s 3 p 2 d / 3 s 2 p+1 s 1 p 1 d$ (this work).

${ }^{\mathrm{d}}$ Original calculations from Ref. 7.

$100 \mathrm{~cm}^{-1}$ upon optimization and comes closer to the observed $0.110 \mathrm{eV}\left(877 \mathrm{~cm}^{-1}\right)$ value. Considering the intensity pattern computed in the present study the assignment clearly corresponds to the fundamental $8_{0}^{1}$.

Finally, the vibrational structure of the $1{ }^{1} A_{1}$ $\rightarrow 2{ }^{1} B_{2}\left(3 p_{x}\right)$ electronic transition has been computed. The overall oscillator strength of this band is half that of the ${ }^{1} B_{1}\left(3 p_{y}\right)$ transition. The maximum intensity is also carried by the $0_{0}^{0}$ excitation, a feature shared by all the transitions to Rydberg states (except in ${ }^{1} A_{2}$ which is one-photon forbidden). The in-plane vibrations carry most of the intense progressions, in particular modes $\nu_{4}$ and $\nu_{5}$, corresponding basically to the bending of the $\mathrm{C}_{1}-\mathrm{C}_{2}-\mathrm{H}_{2}$ angle and the minus combination of the stretchings $\mathrm{N}-\mathrm{C}_{1}$ and $\mathrm{C}_{1}-\mathrm{C}_{2}$. Others, such as the $b_{2}$ modes $\nu_{16}$ and $\nu_{19}$ also contribute. The overall profile of the band is not very different from that obtained for the $1{ }^{1} B_{1}\left(3 p_{y}\right)$ transition. The final assignment of the recorded bands might therefore be questioned if band positions and oscillator strengths were not accurate enough. We cannot assure a high accuracy in the position of the $2{ }^{1} B_{2}\left(3 p_{x}\right)$ band origin, but taking into account the computed intensity, which is much lower for $2{ }^{1} B_{2}\left(3 p_{x}\right)$, and the agreement between theory and experiment in the assignments of the ${ }^{1} B_{1}\left(3 p_{y}\right)$ band, we are confident about the present suggestions.

\section{Valence-Rydberg mixing in the ${ }^{1} B_{2}$ states of pyrrole}

Table $\mathrm{V}$ compiles the computed excitation energies and oscillator strengths for the two low-lying ${ }^{1} B_{2}$ states of pyrrole at different levels of calculation. Two different basis sets and geometries have been employed in order to show the behavior of the CASSCF, CASPT2, and MS-CASPT2 methods. As mentioned in Sec. I, recent high-level $a b$ initio calculations, in particular a MRMP ${ }^{9}$ and a CC approach ${ }^{12}$ obtained vertical excitation energies for the ${ }^{1} B_{2}\left(\pi \pi^{*}\right)$ valence state that differed nearly $0.5 \mathrm{eV}$ from the reported CASPT2 value at $6.00 \mathrm{eV}^{7}$ Additionally, the former methods obtained a larger, although similar, intensity for the ${ }^{1} B_{2}\left(3 p_{x}\right)$ Rydberg than for the valence state. In contrast, the CASSCF/CASPT2 oscillator strengths were computed to be very different for 
the valence $(0.125)$ and the Rydberg (0.040) transition. The present PMCAS-CI/MS-CASPT2 results, performed with a different basis set and geometry, are consistent with the previous CASPT2 description of the pyrrole spectrum, placing an intense intravalence transition inside the $5.5-6.5 \mathrm{eV}$ band. We attribute the discrepancy with the other methods to the presence of a strong valence-Rydberg mixing, which takes place in different regions of the states hypersurfaces and which require specific treatment.

The earlier CASPT2 calculation ${ }^{7}$ employed the experimentally determined ground state geometry. We have used the same geometry to perform CASPT2 and MS-CASPT2 calculations (see Table V) using the original ANO C,N $[4 s 3 p 1 d] / \mathrm{H}[2 s 1 p]+2 s 2 p 2 d$ (basis $A$ ) basis set and the ANO C, N $[4 s 3 p 2 d] / \mathrm{H}[3 s 2 p]+1 s 1 p 1 d$ (basis $B$ ) basis set used in the present study. Seven ${ }^{1} B_{2}$ roots were included in the SA-CASSCF procedure and, for consistency with the previous results, the original (0604) active space was employed for basis $A$, while the present (4523) space was used for basis $B$. The results are very similar in both cases. While the CASPT2 energies place the ${ }^{1} B_{2}\left(\pi \pi^{*}\right)$ valence state above the ${ }^{1} B_{2}\left(3 p_{x}\right)$ Rydberg state, the interaction at the MS-CASPT2 level interchange their positions. Regarding the oscillator strengths, the valence transition carries most of the intensity in all cases, but it is interesting to notice that the CASSCF/CASPT2 oscillator strength drops with basis $B$ to almost half of the previous value. Once the MS-CASPT2 is applied the strength is restored to the valence state. We can conclude that the effect of the MS-CASPT2 is noticeable because the position of the states is interchanged, but their properties remain almost invariant. Therefore, the amount of valence-Rydberg mixing can be said to be small. One of the basic characteristics of large valence-Rydberg mixing is the large change in properties of the involved states. ${ }^{31}$

The performance of the method is different at the CASPT2 ground-state optimized geometry. For basis $A$, although the state ordering remains the same at the CASPT2 level, the excitation energy to the ${ }^{1} B_{2}\left(\pi \pi^{*}\right)$ valence state increases up to $6.88 \mathrm{eV}$, a change of $0.88 \mathrm{eV}$ from the result with the same basis at the experimental geometry. The oscillator strength values, however, do not differ much. The MSCASPT2 treatment again reverses the state ordering, placing the valence state $5.86 \mathrm{eV}$ below the Rydberg state $6.17 \mathrm{eV}$, but the oscillator strength values are now quite different. The Rydberg transition carries more intensity that the valence transition. It is worth mentioning that the differentiation between valence and Rydberg is not clear at this point. Focusing on the orbital extension does not help much because the computed values are similar for both states. We can therefore confirm that at this geometry and using basis $A$ the amount of valence-Rydberg mixing is very large. This is reflected both by the large excitation energy computed at the CASPT2 level and by the undetermined character of the states. Changing to basis $B$ the situation is slightly different. The vertical CASPT2 energy drops to $6.08 \mathrm{eV}$, close to the values obtained at the experimental geometry, but the mixing is this time reflected in the CASSCF oscillator strength value, larger for the Rydberg than for the valence state. The MSCASPT2 treatment is now able to remove the mixing and the finally obtained states at $5.87 \mathrm{eV}$ (valence) and $6.09 \mathrm{eV}$ (Rydberg) have a defined character and corresponding intensities.

Other experiments can be performed with different geometries, basis sets, and active spaces which lead in some cases to heavy mixings, whereas in other situations the states do not strongly interact. The conclusion is that the extent of the mixing is very sensitive to the level of calculation and will depend strongly on the character of the hypersurface where the calculation is performed. We found less mixing at the optimized minima of the states than at the vertical geometries. The MRMP ${ }^{9}$ and $\mathrm{CC}^{12}$ results can therefore be explained in the same context. Müller $e t ~ a l{ }^{45}$ recently showed that a complete removal of the valence-Rydberg mixing problems was not possible even for the most accurate correlation methods such as MS-CASPT2, multireference-singles and doubles configuration interaction (MR-SDCI), multireference quadratic coupled-cluster (MR-AQCC), or equation of motion-coupled-cluster (EOM-CC) in ethene unless the reference wave function is corrected including dynamical correlation effects. We find that MS-CASPT2 can remove most of the mixing ${ }^{25}$ and provide good excitation energies, but in severe valence-Rydberg situations the PMCAS-CI properties can be largely perturbed by the mixing. In our opinion these problems are the main reasons for the discrepancies found in the vertical excitation energies of the ${ }^{1} B_{2}$ states of the pyrrole molecule.

\section{SUMMARY AND CONCLUSIONS}

CASPT2 and MS-CASPT2 calculations on the ground and six low-lying singlet valence and Rydberg excited states of the pyrrole molecule have been performed. The geometries of the states have been optimized by means of the CASPT2 method. Harmonic force fields at the optimized structures were computed at the same level of theory. Vertical and adiabatic excitation energies, together with vertical oscillator strengths, were also obtained. The results were used in the calculation of the vibrational profiles of the electronic absorption bands from the ground to each of the excited states: $\quad 1{ }^{1} A_{2}(3 s), \quad 2{ }^{1} A_{1}\left(\pi \pi^{*}\right), \quad{ }^{1} B_{2}\left(\pi \pi^{*}\right)$, $2{ }^{1} A_{2}\left(3 p_{z}\right), 1{ }^{1} B_{1}\left(3 p_{y}\right)$, and $2{ }^{1} B_{2}\left(3 p_{x}\right)$. The obtained results provide a consistent picture of the recorded spectrum in the energy region 5.5-6.5 eV and confirm that most of the intensity of the low-energy absorption band in pyrrole arises from a $\pi \pi^{*}$ intravalence transition, in contradiction to some recent theoretical conclusions..$^{9,10,12}$ Transition origins and the reported vibrational structure of some bands have been successfully compared with experiments and solved previous uncertainties, while new assignments are tentatively suggested. The discrepancies found between CASPT2 and other methods in the position of the valence $1{ }^{1} B_{2}\left(\pi \pi^{*}\right)$ state are attributed to the large degree of valence-Rydberg mixing present at certain levels of calculation.

\section{ACKNOWLEDGMENTS}

This work has been supported by the Swedish Natural Science Council (NFR), the European Research Training Network THEONET II (Grant No. HPRN-CT-1999-00005), 
the DGES Project No. BQU2001-2926 of Spain, and by the Generalitat Valenciana. B.O.R. acknowledges an IBERDROLA fellowship and V.M. a postdoctoral grant from the MEC of Spain. L.S.A. acknowledges a project in the program "Ramón y Cajal" from the MCYT.

${ }^{1}$ K. Andersson, P.-Å. Malmqvist, B. O. Roos, A. J. Sadlej, and K. Wolinski, J. Phys. Chem. 94, 5483 (1990).

${ }^{2}$ K. Andersson, P.-A. Malmqvist, and B. O. Roos, J. Chem. Phys. 96, 1218 (1992).

${ }^{3}$ B. O. Roos, M. P. Fülscher, P.-Å. Malmqvist, M. Merchán, and L. Serrano-Andrés, in Quantum Mechanical Electronic Structure Calculations with Chemical Accuracy, edited by S. R. Langhoff (Kluwer Academic Dordrecht, The Netherlands, 1995), p. 357.

${ }^{4}$ B. O. Roos, K. Andersson, M. P. Fülscher, P.-A. Malmqvist, L. SerranoAndrés, K. Pierloot, and M. Merchán, in Advances in Chemical Physics: New Methods in Computational Quantum Mechanics, edited by I. Prigogine and S. A. Rice (Wiley, New York, 1996), Vol. XCIII; p. 219.

${ }^{5}$ M. Merchán, L. Serrano-Andrés, M. P. Fülscher, and B. O. Roos, in Recent Advances in Multireference Theory, edited by K. Hirao (World Scientific Singapore, 1999), Vol. IV, p. 161.

${ }^{6}$ B. O. Roos, Acc. Chem. Res. 32, 137 (1999).

${ }^{7}$ L. Serrano-Andrés, M. Merchán, I. Nebot-Gil, B. O. Roos, and M. P. Fülscher, J. Am. Chem. Soc. 115, 6184 (1993).

${ }^{8}$ L. Serrano-Andrés, M. Merchán, M. Fülscher, and B. O. Roos, Chem. Phys. Lett. 211, 125 (1993).

${ }^{9}$ H. Nakano, T. Tsuneda, T. Hashimoto, and K. Hirao, J. Chem. Phys. 104, 2312 (1996)

${ }^{10}$ A. B. Trofimov and J. Schirmer, Chem. Phys. 214, 153 (1997).

${ }^{11}$ M. H. Palmer, I. C. Walker, and M. F. Guest, Chem. Phys. 238, 179 (1998).

${ }^{12}$ O. Christiansen, J. Gauss, J. F. Stanton, and P. Jørgensen, J. Chem. Phys. 111, 525 (1999).

${ }^{13}$ P. A. Mullen and M. K. Orloff, J. Chem. Phys. 51, 2276 (1969).

${ }^{14}$ P. J. Derrick, L. Asbrink, O. Edqvist, B. Ö. Jonsson, and E. Lindholm, Int. J. Mass Spectrom. Ion Phys. 6, 161 (1971).

${ }^{15}$ M. B. Robin, Higher Excited States in Polyatomic Molecules (Academic, New York, 1975), Vol. 2.

${ }^{16}$ M. Bavia, F. Bertinelli, C. Taliani, and C. Zauli, Mol. Phys. 31, 479 (1976).

${ }^{17}$ W. M. Flicker, O. A. Mosher, and A. Kuppermann, J. Chem. Phys. 64, 1315 (1976)

${ }^{18}$ C. D. Cooper, A. D. Williamson, J. C. Miller, and R. N. Compton, J. Chem. Phys. 73, 1527 (1980).
${ }^{19}$ H.-H. Perkampus, C. Schmiele, and T. H. Braunschweig, in UV-VIS Atlas of Organic Compounds, edited by H.-H. Perkampus (VCH, Weinheim, 1992), p. 741

${ }^{20}$ W. Butscher and K. H. Thunemann, Chem. Phys. Lett. 57, 224 (1978).

${ }^{21}$ D. C. Rawlings and E. R. Davidson, Chem. Phys. Lett. 98, 424 (1983).

${ }^{22}$ H. Nakatsuji, O. Kitao, and T. Yonezawa, J. Chem. Phys. 83, 723 (1985).

${ }^{23}$ D. J. Tozer, R. D. Amos, N. C. Handy, B. O. Roos, and L. Serrano-Andrés, Mol. Phys. 97, 859 (1999).

${ }^{24}$ B. O. Roos, K. Andersson, M. P. Fülscher, L. Serrano-Andrés, K. Pierloot, M. Merchán, and V. Molina, J. Mol. Struct.: THEOCHEM 388, 257 (1996).

${ }^{25}$ J. Finley, P.-Å. Malmqvist, B. O. Roos, and L. Serrano-Andrés, Chem. Phys. Lett. 288, 299 (1998).

${ }^{26}$ M. Rubio and B. O. Roos, Mol. Phys. 96, 603 (1999).

${ }^{27}$ R. Crespo, M. Merchán, and J. Michl, J. Phys. Chem. A 104, 8593 (2000).

${ }^{28}$ V. Molina and M. Merchán, J. Phys. Chem. A 105, 3745 (2001).

${ }^{29}$ L. Serrano-Andrés, M. Merchán, A. C. Borin, and J. Stålring, Int. J. Quantum Chem. 84, 181 (2001).

${ }^{30}$ P.-O. Widmark, P.-Å. Malmqvist, and B. O. Roos, Theor. Chim. Acta 77, 291 (1990).

${ }^{31}$ L. Serrano-Andrés, M. Merchán, I. Nebot-Gil, R. Lindh, and B. O. Roos, J. Chem. Phys. 98, 3151 (1993).

${ }^{32}$ P.- $\AA$ Malmqvist, Int. J. Quantum Chem. 30, 479 (1986).

${ }^{33}$ P.-Å. Malmqvist and B. O. Roos, Chem. Phys. Lett. 155, 189 (1989).

${ }^{34}$ A. Bernhardsson, N. Forsberg, P.-Å. Malmqvist, B. O. Roos, and L. Serrano-Andrés, J. Chem. Phys. 112, 2798 (2000).

${ }^{35}$ P.-Å. Malmqvist and N. Forsberg, Chem. Phys. 228, 227 (1998).

${ }^{36}$ L. Serrano-Andrés, N. Forsberg, and P.-A. Malmquist, J. Chem. Phys. 108, 7202 (1998).

${ }^{37} \mathrm{~K}$. Andersson et al., MOLCAS, Version 5.0. Dept. of Theor. Chem., Chem. Center, Univ. of Lund, P.O.B. 124, S-221 00 Lund, Sweden, 2000.

${ }^{38}$ L. Nygaard, J. T. Nielsen, J. Kirchheiner, G. Maltesen, J. RastrupAndersen, and G. O. Sbrensen, J. Mol. Struct. 3, 491 (1969).

${ }^{39}$ C. S. Page (private communication, 2001).

${ }^{40}$ R. McDiarmid and X. Xing, J. Chem. Phys. 105, 867 (1996).

${ }^{41}$ G. Herzberg, Molecular Spectra and Molecular Structure. III. Electronic Spectra and Electronic Structure of Polyatomic Molecules (Van Nostrand, New York, 1966).

${ }^{42}$ J. H. Callomon, T. M. Dunn, and I. M. Mills, Philos. Trans. R. Soc. London, Ser. A 259, 499 (1966).

${ }^{43}$ D. W. Scott, J. Mol. Struct. 37, 77 (1971).

${ }^{44}$ R. C. Lord and F. A. Miller, J. Chem. Phys. 10, 328 (1942).

${ }^{45}$ T. Müller, M. Dallos, and H. Lischka, J. Chem. Phys. 110, 7176 (1999). 